\title{
Basalinsulin und GLP-1-Agonist ergänzen sich
}

\begin{abstract}
Wenn Typ-2-Diabetiker mit einem Basalinsulin plus Metformin nicht mehr gut eingestellt sind, stellt die zusätzliche Gabe eines GLP-1-Agonisten eine wirksame und sinnvolle nächste Stufe der Therapieeskalation dar, so das Ergebnis zweier Studien.
\end{abstract}

- Erst Allgemeinmaßnahmen plus orale Antidiabetika, dann zusätzlich ein Basalinsulin, später auch prandiale Insuline - so lautet der gängige Weg der Therapieeskalation bei Typ-2-Diabetes. Doch der letzte Schritt kann problematisch sein: Werden lang und kurz wirksame Insuline addiert, steigen oft Körpergewicht und Hypoglykämierate weiter an.

\section{Duo attackiert Nüchtern- und postprandiale Glukose}

Eine pathophysiologisch sinnvolle Alternative wäre die Kombination aus Basalinsulin plus GLP-1-Agonist, die beide komplementär agieren. Wie beim USDiabeteskongress präsentierte Studienergebnisse [1] zeigen, senkt Insulin Glargin

v. a. die Nüchtern-Glukose, während der GLP-1-Agonist Lixisenatid überwiegend postprandiale BZ-Spitzen glättet. Wirksamkeit und Verträglichkeit dieser Kombination wurden in zwei Studien untersucht, deren Ergebnisse ebenfalls erstmals beim ADA vorgestellt wurden.

\section{GetGoal-Duo-1-Studie bei} insulinnaiven Patienten

In der GetGoal-Duo-1-Studie [2] wurden 898 insulinnaive Patienten mit im Schnitt neunjähriger Diabetesdauer und suboptimaler Einstellung unter oralen Antidiabetika (OAD) zunächst zwölf Wochen zusätzlich mit Insulin Glargin behandelt. Wie Prof. Julio Rosenstock, Direktor des Diabetes and Endocrine Centers in Dallas berichtete, fielen dadurch die Nüchternglukose-Werte von ca. 190 auf ca. $120 \mathrm{mg} / \mathrm{dl}$ und das $\mathrm{HbA}_{1 \mathrm{c}}$ von 8,6 auf $7,6 \% \mathrm{ab}$.

Anschließend wurde bei 446 Patienten, deren $\mathrm{HbA}_{1 \mathrm{c}}$ weiter zwischen 7 und $9 \%$ lag, Lixisenatid $(1 \times 20 \mu \mathrm{g} / \mathrm{d}) \mathrm{mit}$ Placebo über 24 Wochen doppelblind

\section{Sport und Diät bei Prädiabetes}

\section{Frauen reduzieren langfristig ihr Sterberisiko, Männer nicht}

Mit Lebensstilmodifikationen gelingt es, im Stadium der gestörten Glukosetoleranz (IGT) die Manifestation des Diabetes zu verhindern oder zu verzögern. Ob damit auch ein langfristiger Nutzen verbunden ist, wollten chinesische Wissenschaftlicher wissen, die sechs Jahre lang 576 erwachsene Patienten mit IGT in drei Gruppen behandelt hatten: Mit Diät, mit Bewegung oder mit Diät plus Bewegung. Eine vierte Gruppe diente als Kontrolle.

23 Jahre nach der Randomisierung verglichen die Autoren die Mortalität in der kombinierten Interventionsgruppe und in der Kontrollgruppe, und zwar nach Geschlechtern getrennt.
Dabei zeigte sich, dass Frauen von der Lebensumstellung massiv profitierten: Ihre Gesamtmortalität sank von $29 \%$ auf $16 \%$, v.a. aufgrund einer deutlichen Reduktion der kardiovaskuläre Sterblichkeit von $19 \%$ auf $7 \%$. Das war statistisch hoch signifikant. Männer hingegen strampelten sich vergeblich ab: Maßhalten bei Mahlzeiten nebst Erhöhung der Fitness reduzierte bei ihnen in dieser Studie weder die Gesamtsterblichkeit (41\% vs. $47 \%)$ noch die kardiovaskuläre Sterblichkeit ( $26 \%$ vs. $27 \%$ ).

DE -

- Quelle: 72. Scientific Sessions 2012, American Diabetes Association, Philadelphia, 8.-12. Juni 2012. Li G., et al. Diabetes 2012, Vol. 61, Suppl 1,147 OR, S. A39 verglichen. Der GLP-1-Agonist reduzierte die postprandialen BZ-Werte (zwei Stunden nach Standardfrühstück) gegenüber Placebo um $57 \mathrm{mg} / \mathrm{d}$. Das $\mathrm{HbA}_{1 \mathrm{c}}$ sank unter Lixisenatid von im Schnitt $7,6 \%$ auf $6,96 \%$ und unter Placebo von $7,6 \%$ auf $7,3 \%(\mathrm{p}<0,0001)$. Gleichzeitig reduzierte der GLP-1-Agonist den Insulinbedarf gegenüber Placebo um ca. fünf Units täglich. Den $\mathrm{HbA}_{1 \mathrm{c}}$-Zielwert erreichten 56\% (Lixisenatid) sowie 38,5\% (Placebo).

Lixisenatid reduzierte das Körpergewicht gegenüber Placebo um ca. $1 \mathrm{~kg}$. Die Hypoglykämie-Rate stieg von 0,44 auf 0,8 Episoden pro Jahr. Nur ein Patient erlitt eine schwere Unterzuckerung. $27 \%$ vs. $5 \%$ der Patienten berichteten initial über Übelkeit, $9 \%$ vs. $1 \%$ der Patienten übergaben sich. Die Nebenwirkungen sanken nach acht Wochen auf Placeboniveau.

\section{GetGoal-L-Studie bei mit Insulin vorbehandelten Patienten}

$\mathrm{Zu}$ ähnlichen Ergebnissen kam die doppelblinde GetGoal-L-Studie [3]. Sie untersuchte 495 Patienten mit im Schnitt 12,5-jähriger Diabetesdauer und $\mathrm{HbA}_{1 c^{-}}$ Werten von $8,4 \%$ unter Basalinsulin plus Metformin. Die Ergebnisse: Die $\mathrm{HbA}_{1 c^{-}}$ Werte fielen nach 24 Wochen um 0,74\% unter Lixisenatid bzw. 0,38\% unter Placebo (Differenz 0,36\%, $\mathrm{p}=0,0002$ ); das Körpergewicht sank um 1,8 kg (vs. 0,5 kg unter Placebo), vier Patienten der Verumgruppe (vs. 0) hatten schwere Hypoglykämien.

Lixisenatid (vorgesehenes Warenzeichen: Lyxumia ${ }^{\circ}$ ) ist ein einmal täglich s.c. zu verabreichender GLP-1-Agonist, der von Sanofi klinisch entwickelt wird. Die Zulassung in Europa ist beantragt.

DR. MED. DIRK EINECKE :

- Quelle: 72. Scientific Sessions 2012, American Diabetes Association, Philadelphia, 8.-12. Juni 2012; Diabetes 2012; Vol. 61, Suppl1; 1. 1051-P (S. A 270); 2. 62-OR (S.A18); 3. 983-P (S. A 251) 